\title{
REFINED SYSTEM PARAMETERS AND TTV STUDY OF TRANSITING EXOPLANETARY SYSTEM HAT-P-20
}

\author{
Leilei Sun ${ }^{1,2,3}$, Shenghong Gu ${ }^{1,2,3}$, Xiaobin Wang ${ }^{1,2,3}$, Andrew Collier Cameron ${ }^{4}$, Dongtao Cao ${ }^{1,2,3}$, Yibo Wang ${ }^{1,2,3}$, \\ Yue Xiang ${ }_{1}^{1,2}$, Ho-Keung Hui ${ }^{5}$, Chi-Tai KwoK ${ }^{5}$, Bill Yeung ${ }^{6}$, Eric $\mathrm{NG}^{6}$, and Ferran Grau Horta ${ }^{7}$ \\ ${ }^{1}$ Yunnan Observatories, Chinese Academy of Sciences, Kunming 650011, China; wangxb@ynao.ac.cn \\ ${ }^{2}$ Key Laboratory for the Structure and Evolution of Celestial Objects, Chinese Academy of Sciences, Kunming 650011, China \\ ${ }^{3}$ University of Chinese Academy of Sciences, Beijing 100049, China \\ ${ }^{4}$ School of Physics and Astronomy, University of St Andrews, North Haugh, St Andrews, Fife KY16 9SS, UK \\ ${ }^{5}$ Ho Koon Nature Education cum Astronomical Centre, Sik Sik Yuen, Hong Kong, China \\ ${ }^{6}$ Hong Kong Astronomical Society, Hong Kong, China \\ ${ }^{7}$ Observatori Ca l'Ou, C/de Dalt 18, Sant Martí Sesgueioles, Spain \\ Received 2016 August 18; revised 2016 November 23; accepted 2016 November 23; published 2016 December 28
}

\begin{abstract}
We report new photometric observations of the transiting exoplanetary system HAT-P-20, obtained using CCD cameras at Yunnan Observatories and Ho Koon Nature Education cum Astronomical Centre, China, from 2010 to 2013, and Observatori Ca l'Ou, Sant Marti Sesgueioles, Spain, from 2013 to 2015. The observed data are corrected for systematic errors according to the coarse de-correlation and SYSREM algorithms, so as to enhance the signal of the transit events. In order to consistently model the star spots and transits of this exoplanetary system, we develop a highly efficient tool STMT based on the analytic models of Mandel \& Agol and Montalto et al. The physical parameters of HAT-P-20 are refined by homogeneously analyzing our new data, the radial velocity data, and the earlier photometric data in the literature with the Markov chain Monte Carlo technique. New radii and masses of both host star and planet are larger than those in the discovery paper due to the discrepancy of the radius among K-dwarfs between predicted values by standard stellar models and empirical calibration from observations. Through the analysis of all available mid-transit times calculated with the normal model and spotted model, we conclude that the periodic transit timing variations in these transit events revealed by employing the normal model are probably induced by spot crossing events. From the analysis of the distribution of occulted spots by HAT-P$20 \mathrm{~b}$, we constrain the misaligned architecture between the planetary orbit and the spin of the host star.
\end{abstract}

Key words: planetary systems - stars: individual (HAT-P-20) - techniques: photometric

\section{INTRODUCTION}

Photometric transit surveys have become one of the most successful methods for detecting extrasolar planetary systems over the past decade, yielding 739 planetary systems which contain 372 multiple planet systems as of writing. ${ }^{8}$ Moreover, photometric observations of a transiting system are the only way to directly obtain the size and mass of an exoplanet, combined with radial velocity observations. Follow-up observations for known transiting exoplanetary systems can not only refine the parameters of the system, but also provide important information about the existence of additional bodies in the system with the transit timing variation (TTV) method (Miralda-Escudé 2002; Agol et al. 2005; Holman \& Murray 2005) and/or transit duration variation (TDV) method (Kipping 2009). Through analysis of the long-term monitoring of TTV and/or TDV signals for transiting exoplanetary systems, even based on current ground-based measurements, terrestrial planets become easily detectable, even though they are difficult to detect by other detection methods (Agol et al. 2005; Holman \& Murray 2005). These fundamental parameters are indispensable for the study of planetary properties, such as the composition, structure, and even formation and evolution of the planetary system (Baraffe et al. 2008, 2010; Enoch et al. 2012). Therefore, we have run a monitoring project since 2007 for several known transiting exoplanetary systems by using the 1 and $2.4 \mathrm{~m}$ telescopes of Yunnan Observatories (hereafter, YO-1 m and YO-2.4 m) and the

\footnotetext{
http://exoplanet.eu/catalog/
}

$0.5 \mathrm{~m}$ telescope of Ho Koon Nature Education cum Astronomical Centre (hereafter, HKNEAC-0.5 m) in China, and published a series of observational results (Tan et al. 2013; Wang et al. 2013, 2014; Sun et al. 2015).

However, stellar activity in late-type main-sequence stars induces photometric modulations and apparent radial velocity variations which may hamper the detection of Earth-like planets and the measurement of system parameters, such as the orbital semimajor axis of a planet, the transit depth and so on, which hence biases the inference of radius and mass of both planet and host star, and the characterization of planetary atmospheric properties (Sozzetti et al. 2007; Enoch et al. 2010; Oshagh et al. 2015). Special variations in the spot distribution and the ratio between unperturbed and spotted areas across the stellar surface can modify the mid-transit times when fitted with a normal, symmetric transiting model, due to spot crossing events (Oshagh et al. 2013b; Daassou et al. 2014), which possibly mimic the TTV signals induced by gravitational perturbations of other planets. Therefore, accounting for the effects of stellar activity on transit light curves becomes essential for acquiring accurate physical parameters and appropriately characterizing transiting exoplanetary systems. Based on previous work of Tregloan-Reed et al. $(2013,2015)$ and Montalto et al. (2014), we develop a highly efficient tool by employing the DREAM algorithm (Vrugt et al. 2009) to simultaneously model multiple transiting light curves deformed by spots in the stellar photosphere. Utilizing our new tool, we comprehensively analyze the published and new transit light curves of HAT-P-20. 
Table 1

The Observing Log of HAT-P-20

\begin{tabular}{|c|c|c|c|c|c|}
\hline Date (UT) & Equipment & Filter & Airmass & Exposure Time (s) & $\overline{\mathrm{rms}(\mathrm{mag})^{\mathrm{a}}}$ \\
\hline 2010 Dec 01 & YO-1 m & $\mathrm{R}$ & $1.0-1.2$ & $120-180$ & 0.0025 \\
\hline 2010 Dec 24 & YO-1 m & $\mathrm{R}$ & $1.0-2.1$ & $90-150$ & 0.0024 \\
\hline 2010 Dec 27 & YO-1 m & $\mathrm{R}$ & $1.0-1.2$ & 90 & 0.0018 \\
\hline 2010 Dec 27 & HKNEAC-0.5 m & $\mathrm{R}$ & $1.0-1.14$ & $60-90$ & 0.0021 \\
\hline 2012 Mar 11 & YO-2.4 m & $\mathrm{R}$ & $1.0-1.4$ & 8-20 & 0.0022 \\
\hline 2012 Mar 14 & YO-1 m & $\mathrm{R}$ & $1.0-1.1$ & $60-150$ & 0.0030 \\
\hline 2013 Jan 07 & YO-1 m & $\mathrm{R}$ & $1.1-2.4$ & $120-180$ & 0.0033 \\
\hline 2013 Nov 13 & OCIO-0.3 m & $\mathrm{V}$ & $1.05-1.5$ & 128 & 0.0062 \\
\hline 2013 Dec 06 & OCIO-0.3 m & V & $1.14-1.8$ & 128 & 0.0037 \\
\hline 2014 Oct 24 & OCIO-0.3 m & $\mathrm{R}$ & $1.05-1.4$ & 80 & 0.0024 \\
\hline 2015 Jan 27 & OCIO- $0.3 \mathrm{~m}$ & $\mathrm{R}$ & $1.05-1.5$ & 140 & 0.0042 \\
\hline 2015 Apr 09 & OCIO-0.3 m & $\mathrm{R}$ & $1.14-2.3$ & 180 & 0.0024 \\
\hline
\end{tabular}

Note.

${ }^{\mathrm{a}}$ The root mean square of the residuals between the reduced observational data and the fitting model of the light curve with STMT.

HAT-P-20b was discovered by Bakos et al. (2011). It is a massive hot Jupiter with a mass of $7.246 \pm 0.03 M_{\mathrm{Jup}}$ and a radius of $0.867 \pm 0.033 R_{\mathrm{Jup}}$. Its host star is a $\mathrm{K} 3$ main-sequence star, and it has a relatively strong magnetic activity (Bakos et al. 2011; Shkolnik 2013; Granata et al. 2014; Salz et al. 2015). HAT-P-20 has a red companion (2MASS07273963+2420171, $J-K=0.92$ ) with a $6 . " 925 \pm 0.012$ separation, which is fainter than HAT-P-20 by $\triangle i=2.01 \pm 0.08$ mag (Bakos et al. 2011; Wöllert \& Brandner 2015). Granata et al. (2014) obtained three light curves of HAT-P-20 by utilizing the Asiago $1.82 \mathrm{~m}$ Copernico telescope on 2011 February 5, November 28, and December 22, respectively, and studied the TTV of HAT-P-20 based on their new observations and available data in the literature. Later Baştürk et al. (2015) recalculated the transit parameters of HAT-P-20 relying on their newly obtained transit light curve with the $1 \mathrm{~m}$ Turkish telescope T100 at TUBITAK National Observatory. We have monitored this system at YO since 2010; two partial transit light curves and four complete ones have been obtained. Meanwhile we have acquired a complete transit light curve at HKNEAC in 2010. In addition, we have obtained five light curves at Observatori $\mathrm{Ca}$ I'Ou (hereafter, OCIO) since 2013. Here, we present our results of the transiting system HAT-P-20, based on our new photometric observations, available light curves, and radial velocity curve from the literature (Bakos et al. 2011; Granata et al. 2014; Baştürk et al. 2015). In Section 2, we describe our observations and data reduction techniques. In Section 3, we describe our new tool which is developed to simultaneously model multiple transiting light curves deformed by spots in the stellar photosphere. In Section 5, we estimate the magnetic activity of HAT-P-20 based on archival data. In Section 5, the analysis of all data is performed by employing the Markov chain Monte Carlo (MCMC) technique and its derivatives, and the system parameters are refined. In Section 6, we discuss the new results. Finally, we summarize our study in Section 7.

\section{OBSERVATIONS AND DATA REDUCTION}

\subsection{OBSERVATIONS}

\subsubsection{Photometric Observations with YO-1 m and $\mathrm{YO}-2.4 \mathrm{~m}$ Telescopes}

The transit events of HAT-P-20 were photometrically observed using the Andor $2 \mathrm{~K} \times 2 \mathrm{~K} \mathrm{CCD}$ camera attached to the $1 \mathrm{~m}$ telescope at YO, China, on 2010 December 1, 24, and 27, 2012 March 14, and 2013 January 7. The JohnsonCousins $\mathrm{R}$ filter was used and the field of view was $7.3 \times 7.3 \operatorname{arcmin}^{2}$. The exposure times were appropriately set according to the weather conditions and instrument statuses (see Table 1). Because of the absence of an auto-guiding system in the telescope, the centroid of the target star drifted several pixels on the CCD image from time to time. In order to minimize the systematic error introduced by this issue, we manually adjusted the position of the target star to the initial position.

HAT-P-20 was also photometrically observed using the YFOSC $2 \mathrm{~K} \times 2 \mathrm{~K} C \mathrm{CD}$ camera attached to the $2.4 \mathrm{~m}$ telescope at YO, China, on 2012 March 11 . The field of view was $9.7 \times 9.7 \mathrm{arcmin}^{2}$. During the observing run, the Johnson-Cousins $\mathrm{R}$ filter was used. The weather was photometric.

\subsubsection{Photometric Observation with the HKNEAC-0.5 m Telescope}

On 2010 December 27, HAT-P-20 was observed utilizing the Apogee $3 \mathrm{~K} \times 3 \mathrm{~K} \mathrm{CCD}$ camera attached to the $0.5 \mathrm{~m}$ telescope at HKNEAC, China. During the observations, the Johnson-Cousins R filter was used and the field of view was $31.4 \times 31.4 \operatorname{arcmin}^{2}$. The weather was good for photometric observations.

\subsubsection{Photometric Observation with the OCIO-0.3 $m$ Telescope}

HAT-P-20 was photometrically observed by utilizing the FLI Proline PL1001 $1 \mathrm{~K} \times 1 \mathrm{~K}$ CCD camera attached to the $0.3 \mathrm{~m}$ Meade LX200 ACF telescope at OCIO, Sant Martí Sesgueioles, Spain, on 2013 November 13, December 6, 2014 October 24, 2015 January 27 and April 9. During the first two observations, the FLI RG 50D V filter was used; during the last three observations, the FLI RG 50D R filter was used. The field of view was $29.9 \times 29.9 \operatorname{arcmin}^{2}$. The weather was good for all photometric observations.

\subsection{Data Reduction and Systematic Error Correction}

We implement the data reduction as described by Wang et al. (2013). First, all CCD images are reduced using the IRAF package, including image trimming, bias subtraction, dark 
current subtraction which is only implemented for the observational images of HKNEAC- $0.5 \mathrm{~m}$ telescope and OCIO- $0.3 \mathrm{~m}$ telescope, flat-field correction, and cosmic ray removal. Second, for the calibrated images except the ones from the OCIO- $0.3 \mathrm{~m}$ telescope, the point spread function (PSF) of stars in each image is constructed by utilizing the DAOPHOT sub-package of IRAF based on the isolated, bright stars in the images and then is used to remove the flux of the companion of HAT-P-20 (Stetson 1987). Third, values of instrumental magnitude for the target star and reference stars are measured with the aperture photometry method using the APPHOT sub-package of IRAF. In the measurements, we have tried a series of apertures to conduct the aperture photometry. Then the optimal transit light curves are obtained.

The transit signal of an exoplanet is normally small. In order to enhance the signal of transit events (Tamuz et al. 2005; Collier Cameron et al. 2006; Wang et al. 2013), we correct the systematic errors in the photometric data based on the coarse decorrelation and SYSREM algorithm. However, there still are several systematic trends left in the transit light curves because of differing extinction between target and reference stars and even the flux modulation induced by starspots on the stellar surface of the rotation of the host star. So we use a linear or quadratic function to fit the out-of-transit data for the trend and remove it from the light curve. Finally, we convert the local observing time into the barycentric Julian date under Barycentric Dynamical Time (BJD ${ }_{\mathrm{TDB}}$ ) (Eastman et al. 2010). After finishing the above procedure, we derive the final light curves of the transit events of HAT-P-20, which are used to analyze the spot structure and the physical parameters of the system in Section 5.

\section{SPOT AND TRANSIT MODELING TOOL (STMT)}

Photometric modulation induced by stellar activities can also appear in the optical light curves of transit events when the host stars are magnetically active. This effects can: (1) modify the total flux of the visible stellar surface and hence change the entire shape of the transit light curve when normalized to the out-of-transit flux (Oshagh et al. 2013b; Daassou et al. 2014; Herrero et al. 2015); in the case of short transit duration (namely, transit duration $T_{14} \ll$ stellar rotational period $P_{\text {rot }}$ ) and no dramatic transient events, like the eruption of an optical flare, the shape of a transit light curve which is not distorted by other systematic noises is still symmetric like the transit light curve of a quiet star; and (2) modify the local shape of the transit light curve while the planetary disc overlays the spot and/or faculae on the stellar disc during the planet transit, namely spot crossing events. The distribution and size of spots occulted by the planet can be obtained precisely through fitting the anomalies of high-precision transit light curves induced by the spot crossing events (Silva 2003; Pont et al. 2007; SilvaValio 2008; Désert et al. 2011; Nutzman et al. 2011; SanchisOjeda et al. 2011, 2013; Tregloan-Reed et al. 2013, 2015). Hence, even when a symmetric light curve modified by several un-occulted spots on the stellar surface is fitted with the theoretical model of the transit event, most transiting parameters directly characterizing the properties of the system cannot be obtained accurately and then the physical parameters of the system deduced from these transit parameters are biased, such as the orbital inclination of the planet, the orbital semimajor axis of the planet, and even the radii and mass of both the host star and planet. Furthermore, the mid-transit times will be biased when the spot crossing events occur during ingress and egress processes of transit events, which will likely mimic the TTV signals induced by the gravitational perturbation of planets (Barros et al. 2013).

The significant influences due to starspots on transit light curves and lots of photometric observations of transit anomalies motivate the development of astrophysical models for analyzing light curves of planetary transits when there are starspots on the stellar disc. Examples include the model by Silva (2003), the model by Wolter et al. (2009), SOAP-T by Oshagh et al. (2013a), PRISM by Tregloan-Reed et al. (2013), SPOTROD by Béky et al. (2014), KSint by Montalto et al. (2014), and ELLC by Maxted (2016). These models, except those of Montalto et al. (2014) and Maxted (2016), numerically integrate over the stellar surface or projected disc to calculate the transit light curve. Montalto et al. (2014) have presented the analytic solution to the stellar spot problem with a quadratic limb-darkening law by employing the Kelvin-Stokes theorem (Pál 2012). Here, we present a highly efficient STMT by employing a modified KSint and Mandel \& Agol (2002)'s Occultquad model, GEMC, and DREAM algorithm proposed respectively by Tregloan-Reed et al. (2013) and Vrugt et al. (2009) as its main building blocks so as to analyze light curves of planetary transits with spots on the visible stellar surface.

\subsection{Modified KSint and Occultquad Model}

To improve the efficiency of modeling the stellar spots and transit events, Montalto et al. (2014) developed an analytic model KSint based on the idea proposed by Pál (2012), which applies the Kelvin-Stokes theorem to the occulting region (e.g., the spotted region in the case of a starspot) and thereby performs the integration only over the border rather than over the entire surface of the region. KSint has been demonstrated to be much more efficient than direct integration when dealing with complex geometries, and still preserves the accuracy of the solution in general (Montalto et al. 2014).

Nonetheless, the entire modification of the transit light curve is only partially attributed to the non-occulted spots in the photosphere because the occulted spots also play the same role in the modification, although the properties of occulted spots by the planet can be diagnosed by modeling transiting data with sufficiently high precision and cadence (Tregloan-Reed et al. 2013). Meanwhile, the transit light curve imposes no more constraints on the properties of non-occulted spots than total fractional deficit of stellar flux when only a single, shortobserving-window transit light curve is available for each transit event, which is ubiquitous in ground-based observation of transit events of exoplanetary systems. It has been known that inverting a rotational modulation light curve to derive spot geometries and distributions is in fact a very complex problem which in general does not lead to a unique solution, because there exists a high degree of degeneracy among the parameters characterizing spots and the orientation of the stellar spin (Eker 1999; Montalto et al. 2014). As a consequence, laying down occulted spots and non-occulted spots simultaneously in the stellar photosphere will not be an ideal way to reproduce these transit light curves deformed by starspots. We therefore adapt the original KSint model by replacing the procedure of laying down real non-occulted spots in the photosphere by fitting a free parameter $\xi_{\text {non }}$ to represent the contribution of nonocculted spots to the fractional deficit of stellar flux. We also refine several numerical algorithms in KSint to eliminate almost entirely the noise generated by publicly available KSint 
code, which contain those demonstrated in Maxted (2016). Meanwhile, Mandel \& Agol (2002)'s analytic Occultquad model is modified by adding the parameter $\xi_{\text {non }}$ to model highly symmetric transit light curves which are influenced by only non-occulted spots. In addition, we define the location of spots on the stellar surface in the reference system in which the spin of the host star is to both perpendicular to the line of sight and the transiting trajectory on the stellar disc, as in Tregloan-Reed et al. (2013), in order to elucidate the degeneracies between the parameters characterizing the spot properties and the orientation of stellar spin axis. We implement both adapted models in STMT to simulate the transit light curves of the spotted star.

\subsection{Parameter Optimization and Estimation Algorithm}

Besides the implementation of the modified analytic KSint and the Occultquad model, STMT is endowed with the ability of simultaneously fitting multiple transit light curves of a spotted star so as to more accurately derive transit parameters and spot properties on the stellar surface. This configuration requires a highly efficient algorithm to estimate the parameters of spots and transits since these are also generally highly correlated besides the mutual correlation among the parameters characterizing the spot properties, even multi-modal distributions and thus high-dimensional when many transit light curves are jointly modeled. For example, assuming $N_{t}$ transit light curves to be fitted jointly, STMT will need $5+4 \sum_{i=1}^{N_{t}}\left(N_{\mathrm{occ}}^{i}+1\right)$ free parameters to characterize these (see Section 5 for more details), where $N_{t}$ and $N_{\text {occ }}^{i}$ respectively denote the total number of transit light curves and occulted spots by the planet in the $i$ th transit event.

Tregloan-Reed et al. (2013) developed an efficient optimization algorithm the so-called genetic evolution Markov chain (GEMC) - by combining the superior global optimization power of genetic algorithms (Charbonneau 1995) with the capability of parameter estimation of differential evolution Markov chain (DE-MC) proposed by ter Braak (2006) for simulating high-dimension, multi-modal systems (Gregory 2011; Eastman et al. 2013; Cubillos et al. 2014). Because GEMC mimics biological processes by spawning successive generations of solutions based on breeding and mutation operations from the parent generation population, the new proposals of Markov chains generated by GEMC based on the fitness of the parent generation population rather than tentatively perturbing the individual parent generation by most MCMC algorithms, thus could jump large distances across the solution space and find where the global solution is (TregloanReed et al. 2013). Therefore, we take advantage of the capability of global exploration of GEMC in STMT.

In order to improve the efficiency of MCMC simulation, Vrugt et al. (2009) proposed a self-adaptive randomized subspace sampling algorithm DREAM based on the DE-MC algorithm of ter Braak (2006). The applicability of DREAM to complex, multi-modal search problems is generally superior to most MCMC sampling approaches because this scheme runs multiple different chains simultaneously for global exploration, and automatically tunes the scale and orientation of the proposal distribution in randomized subspace sampling (Vrugt et al. 2009).

Although the original version of ter Braak (2006)'s DE-MC algorithm has been widely applied to study exoplanetary systems (Gregory 2011; Eastman et al. 2013; Cubillos et al. 2014), DREAM has not yet been utilized in the field (details can be found in Vrugt et al. 2009; Laloy \& Vrugt 2012). The DREAM algorithm has inherited mostly good properties of the DE-MC algorithm, such as the ability of automatically selecting an appropriate scale and orientation for proposal distribution en route to the target distribution (ter Braak 2006; Vrugt et al. 2009). Furthermore, the statistical average of many pairs of parent generations in the DREAM algorithm increases the diversity of the exploration of the parameter space; the scheme of randomized subspace sampling of the DREAM algorithm potentially increases the acceptance of the proposal point for high-dimension, highly correlated systems. The efficiency of the DREAM sampler has been well tested by numerical experiments and simulations of real data (Vrugt et al. 2009; Laloy \& Vrugt 2012). We have tested STMT with the observational data of WASP-19 by Tregloan-Reed et al. (2013, 2015) and found that not only transit light curves simulated by STMT and PRISM agree with each other very well in the majority of situations ${ }^{9}$, but also the ability of both global optimization and parameter estimation of STMT is quite efficient. In the following sections, the practicality of STMT will be enhanced and further discussed.

\section{THE MAGNETIC ACTIVITY OF HAT-P-20}

Before analyzing the transit light curves with STMT, we estimate the photospheric activity of HAT-P-20 by calculating the generalized Lomb-Scargle (GLS) periodogram (Zechmeister \& Kuerster 2009) of the HATNet survey data ${ }^{10}$ and the WASP survey data ${ }^{11}$ of HAT-P-20. We find that optically variable modulation exists in both survey data which is probably due to photospheric spots which persisted at fixed longitudes of HAT-P-20 for a long time, which is consistent with strong chromospheric $\mathrm{Ca}$ II $\mathrm{H} \& \mathrm{~K}$ emission and $\mathrm{X}$-ray emission (Bakos et al. 2011; Granata et al. 2014; Salz et al. 2015), and both peak powers overlay quite well in the GLS periodogram (e.g., $\quad P_{\text {rot }}=14.66 \pm 0.03$ day; $\quad$ see Figure 7).

Unfortunately, there is no long-term continuous highprecision time-series photometric data of HAT-P-20 during the span of all available transit observations described in the following section to be used to correct the flux modulation in these transit light curves for accurately measuring the transit parameters. Nonetheless, based on the reduced spectra of HATP-20 retrieved from the Keck archive, which were observed in the span of these transit observations, we will use the chromospheric activity of the host star to estimate the photospheric activity level because chromospheric activity is generally correlated with photospheric activity for most active late-type main-sequence stars (Berdyugina 2005). Through comparing the average levels of chromospheric activity, we can estimate the relative photospheric activity to some degree besides checking the features of magnetic activity in the chromosphere. Ca II H\&K lines (3968.5 and $3933.7 \AA$ ) are two indicators of stellar chromospheric activity (Wilson 1968), so we select $\mathrm{Ca}$ II $\mathrm{H} \& \mathrm{~K}$ emission originating from the chromosphere as tracers of chromospheric activity. However, it is quite difficult to fit a consistent continuum in these spectral

\footnotetext{
9 In some special cases, for example, the simulated spots cover over $70 \%$ of the stellar surface, PRISM produces a lot of numerical noise since this numerical algorithm deals with the integral of the proximity of the spotted region's boundary imperfectly.

${ }^{10} \mathrm{http}: / /$ hatnet.org/

11 http://www.superwasp.org/
} 


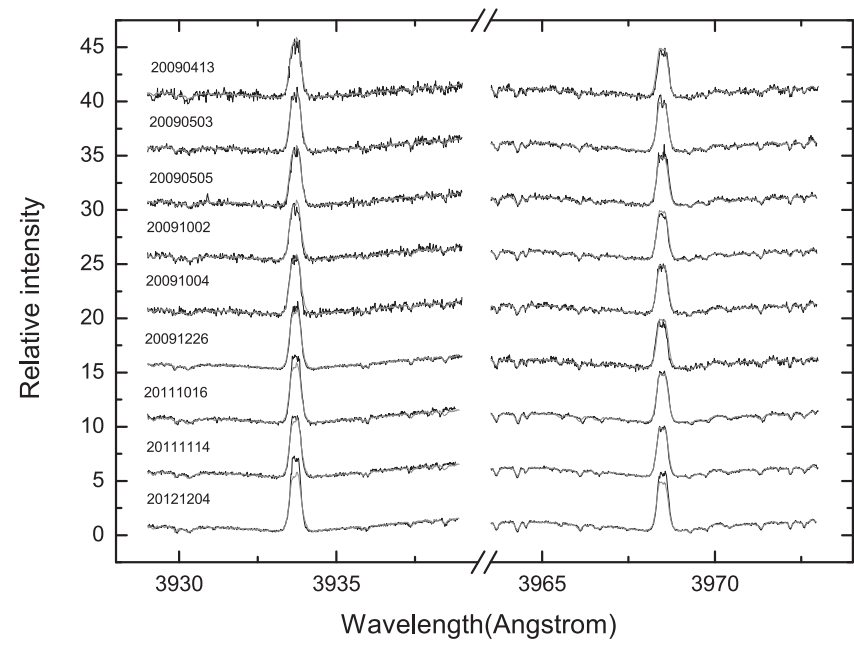

Figure 1. The Ca II H (right) and Ca II K (left) lines of HAT-P-20 from 2009 to 2012, the dashed lines overlayed on the spectra represent the overall average of both spectra.

regions, so we choose narrow bandpasses centered on these two lines, which sets a normalization level as described by Shkolnik et al. (2003, 2005). Portions of the spectra, each $7 \AA$ wide, were extracted centered on $\mathrm{Ca}$ II $\mathrm{H}$ and $\mathrm{Ca}$ II $\mathrm{K}$. When normalizing each sub-spectrum, the edges were set to unity and fitted with a straight line. An overall average was then taken over all nights (e.g., the dashed lines in Figure 1), from which we estimate relative levels of chromospheric activity. The chromospheric activity varies among different rotational phases, and the levels of both lines in 2011 and 2012 are generally stronger than those of 2009 (see Figure 1). Therefore, we ascertain that the levels of stellar magnetic activity of HATP-20 during two transit events observed in 2009 (Bakos et al. 2011) are the lowest, and probably fewer spots appear on the stellar surface, which means that these two light curves can be selected as fiducial light curves for the measurement of initial transit parameters, due to the consistency of lower average levels of chromospheric activity in 2009.

\section{LIGHT CURVE ANALYSIS}

In the analysis of light curves, we have employed the MCMC technique of Collier Cameron et al. (2007) beside the application of STMT. The calibration of Enoch et al. (2010) is utilized in the code to infer the mass of the host star (Sun et al. 2015). The light curves are modeled based on the small planet approximation model (Mandel \& Agol 2002) and the four-coefficient limb-darkening law is used. Four limbdarkening coefficients are derived through interpolation of the coefficient tables of Claret (2000, 2004). However, the quadratic limb-darkening law is used when transit light curves are modeled with STMT. The best fitting values and uncertainties of the system parameters are inferred from their posterior probability distributions.

Here, we describe the fitting scheme and free parameters in STMT. STMT derives the transit parameters and spot properties by modeling simultaneously multiple transit light curves distorted by starspots by employing the GEMC and DREAM algorithms. It can account for the eccentric and circular architecture of a planetary orbit by enforcing a Gaussian penalty of eccentricity $e$ and argument of periastron $\omega$ of planetary orbit. There are $5+4 \sum_{i=1}^{N_{t}}\left(N_{\mathrm{occ}}^{i}+1\right)$ free parameters to characterize all light curves, which depend on the total number of transit light curves and the total number of occulted spots by the planet during each transit event. As described in the previous section, STMT has five common transit parameters which include radii ratio $\frac{R_{p}}{R_{*}}$ between planet and host star, the sum of the fractional radii $\frac{R_{p}+R_{*}}{a}$ with planet and host star normalized to the semimajor axis $a$ of the planetary orbit, the orbital inclination of the planet $i$, and the eccentricity $e$ and argument of periastron $\omega$ of the planetary orbit to characterize the transiting exoplantary system. For a specified light curve, the STMT needs the mid-transit time $T_{c}$ of the transit event, the fractional deficit of the stellar flux $\xi_{\text {non }}$ due to non-occulted spots on the stellar surface, the linear limbdarkening coefficient $u_{1}$ and the quadratic limb-darkening coefficient $u_{2}$ of the standard quadratic limb darkening law, and multiple pairs of longitude of occulted spots' center $\theta$, colatitude of occulted spots' center $\phi$, size of occulted spots $r_{\text {spot }}$, and contrast $\rho_{\text {spot }}$ of occulted spots to the surrounding photosphere, besides the five common transit parameters.

\subsection{Initial Analysis}

Besides our own light curves, we also collect two Sloan $i$ band light curves from Bakos et al. (2011), three $R$-band light curves from Granata et al. (2014), and one $R$-band light curve from Baştürk et al. (2015). Meanwhile, we have obtained raw photometric images of three transit events sent to ETD $^{12}$ by Paul Benni, Alberto Villa, and Mark Salisbury, and then reduced these data with our standard procedure. In addition, we have collected 13 light curves of transit events with moderately high precision and symmetric geometry. All analyses are based on these 34 light curves and radial velocity curve collected from Bakos et al. (2011).

It should be noted that a fiducial light curve is indispensable for the reliable measurement of transit parameters and spot properties by fitting either a single transit light curve or multiple ones simultaneously which are distorted by nonocculted spots on the stellar surface, because both transit parameters and spot properties are inferred from the shape of the transit light curve, and the shape of a transit light curve distorted by spots is defined as the product of a standard transit light curve multiplied with a scale factor $\frac{1}{1-\xi_{\text {non }}}$ for the case without a spot crossing event. This implies high degeneracies between the transit parameters and $\xi_{\text {non }}$ characterizing nonocculted spots. A similar degeneracy also appears in the case of spot crossing events. The correlation has been demonstrated in the following results by employing STMT to simultaneously fit three high-precision, symmetric transit light curves with all $\xi_{\text {non }}$ being set as free parameters. (1) There is an intense instability of the posterior probability distributions of transit parameters and $\xi_{\text {non }} ;(2)$ there exists a high correlation, particularly in the joint posterior probability distribution, between $\frac{R_{p}}{R_{*}}$ and $\xi_{\text {non }}$ (see Figure 2).

Therefore, for the initial analyses of transit light curves using STMT, we simultaneously model four high-precision light curves that are highly symmetric or weakly distorted by occulted spots with all other $\xi_{\text {non }}$ set as free parameters. Bakos et al. (2011)'s light curves observed in 2009 are set as zero to be supplied as fiducial transit light curves, which allows $\xi_{\text {non }}$ to

\footnotetext{
${ }^{12}$ http://var.astro.cz/ETD
} 


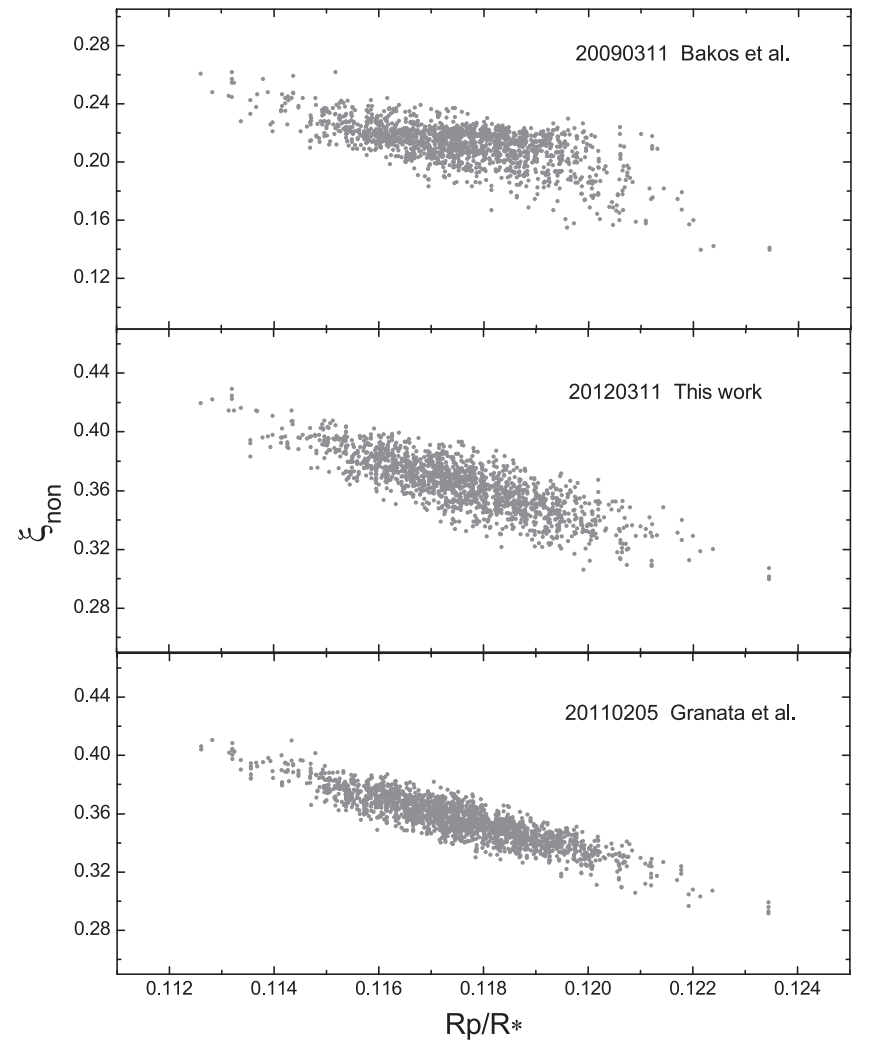

Figure 2. Joint posterior probability distributions of fractional flux of HAT-P$20 \xi_{\text {non }}$ blocked by non-occulted starspots and radius ratio between planet and host star when modeling by employing STMT with all $\xi_{\text {non }}$ being set as free parameters.

absorb the flux modulation induced by non-occulted spots, so as to derive accurate initial values of transit parameters. These four light curves include the two $i$-band light curves of Bakos et al. (2011), our new observation with the YO-2.4 m telescope, and the light curve observed by Baştürk et al. (2015). We derive initial values of transit parameters through 20 times simultaneous modeling of these four transit light curves by utilizing STMT to generate 60,000 samples, $20 \%$ of which are burn-in samples and eliminated in the statistics of posterior probability distribution. During the modeling, the orbital period of HAT-P-20b is fixed to the value derived by Granata et al. (2014), and the search intervals of $u_{1}$ and $u_{2}$ are set to $\left(u_{1 c}-0.05, u_{1 c}+0.05\right)$ and $(0.1,0.3)$, respectively, where $u_{1 c}$ denotes the values interpolated from Claret (2000, 2004)'s table based on the stellar parameters derived by Bakos et al. (2011). The Gaussian penalties of $e$ and $\omega$ are set to the best estimations and uncertainties of Deming et al. (2015) derived from simultaneously modeling transits, secondary eclipses, and radial velocity data of HAT-P-20, through which the most accurate $e$ and $\omega$ could be obtained. We check the posterior probability distributions of several main transit parameters and confirm the convergence of the solution through the fact that the majority of these models have similar distributions for these parameters. Finally, the optimal solution is inferred jointly from the modeling.

\subsection{The Mid-transit Times of HAT-P-2Ob}

In order to study the effect of starspots on the mid-transit times of these transit light curves and to examine whether
Table 2

The Mid-transit Times of HAT-P-20b Based on the Normal Model

\begin{tabular}{|c|c|c|c|c|}
\hline Cycle & $\begin{array}{c}\mathrm{BJD}_{\mathrm{TDB}} \\
-2450000\end{array}$ & $\begin{array}{l}\text { Uncertainty } \\
\max \left(\sigma_{+}, \sigma_{-}\right)\end{array}$ & $(O-C)(\mathrm{d})$ & Source \\
\hline-353 & 4902.657610 & 0.000220 & -0.000204 & Bakos et al. \\
\hline-275 & 5126.932450 & 0.000200 & -0.000109 & Bakos et al. \\
\hline-134 & 5532.351257 & 0.000420 & -0.001035 & This work \\
\hline-126 & 5555.355450 & 0.001282 & 0.000620 & This work \\
\hline-125 & 5558.231145 & 0.000249 & 0.000998 & This work \\
\hline-125 & 5558.228982 & 0.000348 & -0.001165 & This work \\
\hline-121 & 5569.732207 & 0.000470 & 0.000791 & ETD \\
\hline-119 & 5575.480344 & 0.000230 & -0.001707 & ETD \\
\hline-111 & 5598.484247 & 0.000400 & -0.000341 & ETD \\
\hline-111 & 5598.485240 & 0.000200 & 0.000651 & Granata et al. \\
\hline-110 & 5601.360083 & 0.000420 & 0.000177 & ETD \\
\hline-106 & 5612.861543 & 0.000700 & 0.000368 & ETD \\
\hline-8 & 5894.644000 & 0.000490 & 0.001734 & Granata et al. \\
\hline-8 & 5894.642974 & 0.000490 & 0.000709 & ETD \\
\hline-7 & 5897.518646 & 0.000490 & 0.001063 & ETD \\
\hline 0 & 5917.644440 & 0.000430 & -0.000364 & Granata et al. \\
\hline 18 & 5969.401528 & 0.000560 & 0.001014 & ETD \\
\hline 28 & 5998.153904 & 0.000380 & 0.000218 & This work \\
\hline 29 & 6001.029470 & 0.000945 & 0.000466 & This work \\
\hline 110 & 6233.929126 & 0.000420 & -0.000575 & ETD \\
\hline 129 & 6288.560904 & 0.000550 & 0.000175 & ETD \\
\hline 239 & 6604.846419 & 0.000300 & 0.000793 & ETD \\
\hline 241 & 6610.596190 & 0.000760 & -0.000071 & This work \\
\hline 249 & 6633.600233 & 0.000340 & 0.001434 & This work \\
\hline 275 & 6708.356077 & 0.000196 & -0.000971 & Baştürk et al. \\
\hline 283 & 6731.358817 & 0.000386 & -0.000769 & ETD \\
\hline 283 & 6731.361311 & 0.000490 & 0.001725 & ETD \\
\hline 361 & 6955.633993 & 0.000400 & -0.000339 & This work \\
\hline 370 & 6981.510756 & 0.000430 & -0.001430 & ETD \\
\hline 394 & 7050.519975 & 0.000654 & 0.000174 & This work \\
\hline 395 & 7053.394751 & 0.000370 & -0.000367 & ETD \\
\hline 419 & 7122.402932 & 0.000654 & 0.000200 & This work \\
\hline 497 & 7346.678211 & 0.000490 & 0.000733 & ETD \\
\hline
\end{tabular}

TTVs exist in the transiting exoplanet system HAT-P-20, we analyze the light curves with two models. One is the normal model of the transit light curve with no consideration of distortion by starspots; the other is the spotted model STMT which considers the distortion of starspots on the transit light curve.

For the analysis of transit light curves with the no-spot model, we first intended to homogeneously fit these light curves using STMT in the no-spot mode, but we find that our fitting results for mid-transit times of several light curves from ETD, Bakos et al. (2011), and Granata et al. (2014) are in good agreement with their results. Although the majority of the fitting parameters of these models in the literature and ETD are different from each other, except for the mid-transit times, the mid-transit time only depends on the symmetry of the transit light curve. These fitting results of mid-transit times derived from different models should be consistent with each other. Therefore, we fit our new observations with STMT and adopt the best fitting results of others from the literature and ETD. Mid-transit times calculated by the normal model are listed in Table 2.

Subsequently, we individually model each light curve by employing STMT with $\frac{R_{p}}{R_{*}}, \frac{R_{p}+R_{*}}{a}$, and $i$ fixed to the above best initial values so as to efficiently derive the properties of spots on the stellar disc when the transit occurs. We derive the 


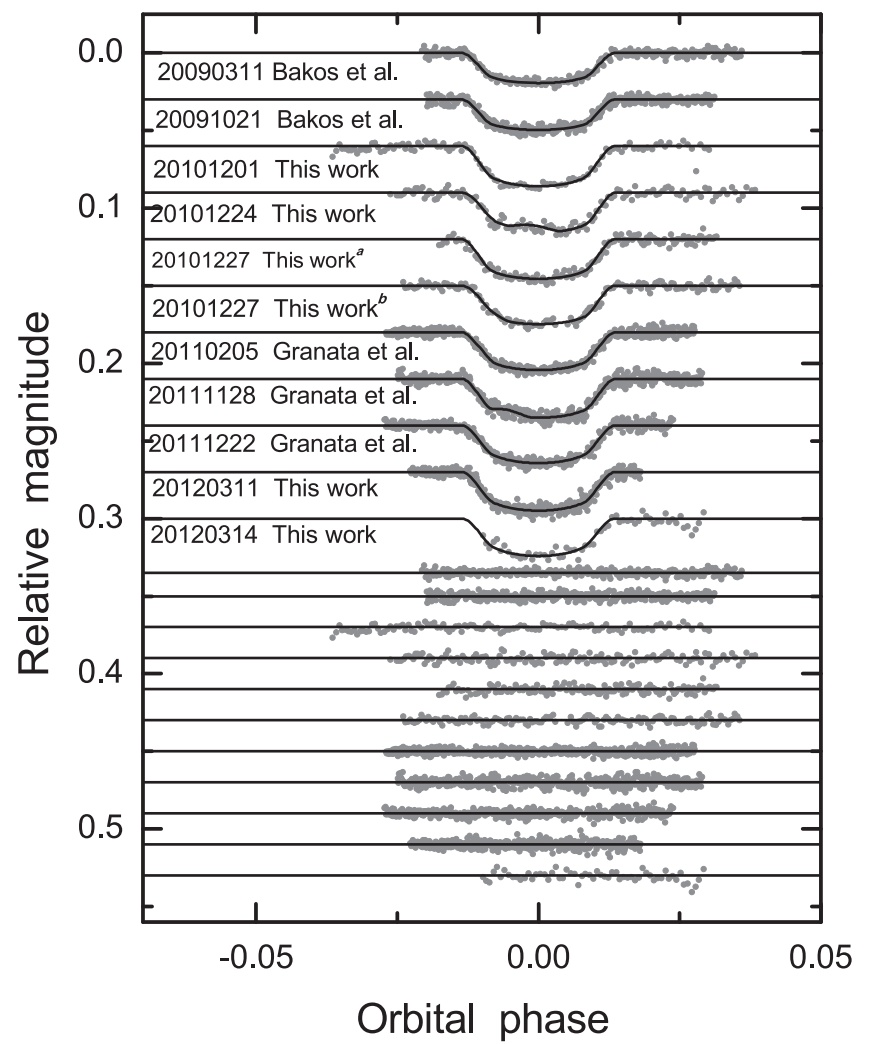

Figure 3. Model fittings of transit light curves of HAT-P-20 with STMT and relative residuals, where superscripts $a$ and $b$ represent the observations at HKNEAC and YO on 2010 December 27, respectively.

optimal fitting result through modeling each transit light curve five times and jointly analyzing the posterior probability distributions of spot parameters. Through the modeling with STMT, the influences of spots and even the systematic flux distortions on each transit light curve induced by the nearby companion along with the variation of seeing are properly removed, and light curves potentially characterizing these transit events are extracted based on the best fitting results. As implemented in the following subsection, the physical parameters of the transiting exoplanetary system HAT-P-20 can be derived based on these potential light curves by utilizing the MCMC technique of Collier Cameron et al. (2007). The best fitting results of these transit light curves are shown in Figures 3-5, respectively. All mid-transit times calculated with STMT are listed in Table 3. Spot parameters derived with STMT are tabulated in Table 4.

We use a linear ephemeris formula $T=T_{0}+P \times E$ to fit these two groups of mid-transit times so as to derive the orbital period values individually, where $T_{0}$ indicates the zero point, $E$ is the orbital cycle, and $P$ is the orbital period. Both $(O-C)$ diagrams are shown in Figure 6. For mid-transit times calculated with the normal model, the ephemeris is $T\left(\mathrm{BJD}_{\mathrm{TDB}}\right)=5917.644804(61)+2.87531725(26) \times E$ and the reduced $\chi^{2}$ is 8.14 . The rms of the $(O-C)$ diagram with the normal model reaches up to 88 seconds when a linear relation is fitted. We have searched for significant, periodic signals by calculating the GLS periodogram and found that the peak power of $(O-C)$ falls down to the zone of the rotational period of HAT-P-20 (e.g., the shaded zone in Figure 7). The reduced $\chi^{2}$ of the $(O-C)$ diagram is decreased to 4.67 when this
Table 3

Mid-transit Times of HAT-P-20b Based on the Spotted Model

\begin{tabular}{|c|c|c|c|c|}
\hline Cycle & $\begin{array}{c}\mathrm{BJD}_{\mathrm{TDB}} \\
-2450000\end{array}$ & $\begin{array}{l}\text { Uncertainty } \\
\max \left(\sigma_{+}, \sigma_{-}\right)\end{array}$ & $(O-C)(\mathrm{d})$ & Source \\
\hline-353 & 4902.657511 & 0.000213 & 0.000158 & Bakos et al. \\
\hline-275 & 5126.932315 & 0.000248 & 0.000222 & Bakos et al. \\
\hline-134 & 5532.351228 & 0.000417 & -0.000584 & This work \\
\hline-126 & 5555.353765 & 0.000437 & -0.000585 & This work \\
\hline-125 & 5558.229276 & 0.000321 & -0.000391 & This work \\
\hline-125 & 5558.230014 & 0.000392 & 0.000347 & This work \\
\hline-121 & 5569.731298 & 0.000611 & 0.000362 & ETD \\
\hline-119 & 5575.480152 & 0.000285 & -0.001418 & ETD \\
\hline-111 & 5598.484304 & 0.000444 & 0.000197 & ETD \\
\hline-111 & 5598.484272 & 0.000233 & 0.000165 & Granata et al \\
\hline-110 & 5601.359837 & 0.000418 & 0.000413 & ETD \\
\hline-106 & 5612.861242 & 0.000769 & 0.000549 & ETD \\
\hline-8 & 5894.642779 & 0.000248 & 0.001004 & Granata et al \\
\hline-8 & 5894.642807 & 0.000508 & 0.001032 & ETD \\
\hline-7 & 5897.517774 & 0.000601 & 0.000682 & ETD \\
\hline 0 & 5917.643372 & 0.000176 & -0.00094 & Granata et al \\
\hline 18 & 5969.400640 & 0.000871 & 0.000619 & ETD \\
\hline 28 & 5998.154095 & 0.000212 & 0.000902 & This work \\
\hline 29 & 6001.029184 & 0.000968 & 0.000674 & This work \\
\hline 110 & 6233.929544 & 0.000612 & 0.000344 & ETD \\
\hline 129 & 6288.560818 & 0.000554 & 0.000592 & ETD \\
\hline 239 & 6604.845985 & 0.000552 & 0.000870 & ETD \\
\hline 241 & 6610.595539 & 0.000648 & -0.000210 & This work \\
\hline 249 & 6633.599465 & 0.000607 & 0.001179 & This work \\
\hline 275 & 6708.356055 & 0.000156 & -0.000477 & Baştürk et al. \\
\hline 283 & 6731.359044 & 0.000683 & -0.000026 & ETD \\
\hline 283 & 6731.358709 & 0.000388 & -0.000361 & ETD \\
\hline 361 & 6955.633811 & 0.001015 & 0.000003 & This work \\
\hline 370 & 6981.511513 & 0.000781 & -0.00015 & ETD \\
\hline 394 & 7050.520041 & 0.000684 & 0.000766 & This work \\
\hline 395 & 7053.395300 & 0.000594 & 0.000708 & ETD \\
\hline 419 & 7122.403120 & 0.000553 & 0.000916 & This work \\
\hline 497 & 7346.677764 & 0.000554 & 0.000821 & ETD \\
\hline
\end{tabular}

periodic signal is removed by fitting with a sinusoidal curve. We suspect that this periodic signal in the $(O-C)$ diagram is not a TTV signal induced by gravitational perturbation from other non-detected bodies in the transiting system. It is probably induced by spot crossing events along with the rotational modulation of spots on HAT-P-20. For mid-transit times calculated with STMT, the derived ephemeris is $T$ $\left(\mathrm{BJD}_{\mathrm{TDB}}\right)=5917.644312(61)+2.87531716(29) \times E$ and the reduced $\chi^{2}$ is 4.09 . We have also searched for significant, periodic signals by calculating the GLS periodogram, but no significant signal is detected. The GLS power resembles a periodogram of random noise. So we confirm that the influences of starspots on these mid-transit times are well depressed when the transit light curves distorted by starspots are appropriately fitted with STMT, as compared with the GLS power periodogram of the $(O-C)$ diagram based on the midtransit times calculated with the normal model. We conclude that the periodic signal in the $(O-C)$ diagram based on the normal model is induced by spot crossing events along with the rotational modulation of spots on HAT-P-20. Furthermore, we find that the orbital period of both linear ephemeris are consistent with each other, but both zero points show a deviation (e.g., $\Delta T_{0}=4.92 \times 10^{-4}$ (day)). The significance level of these deviations reaches $8 \sigma$. So there are no apparent, 
Table 4

Spot Parameters Derived from Modeling Transit Light Curves of HAT-P-20b with STMT

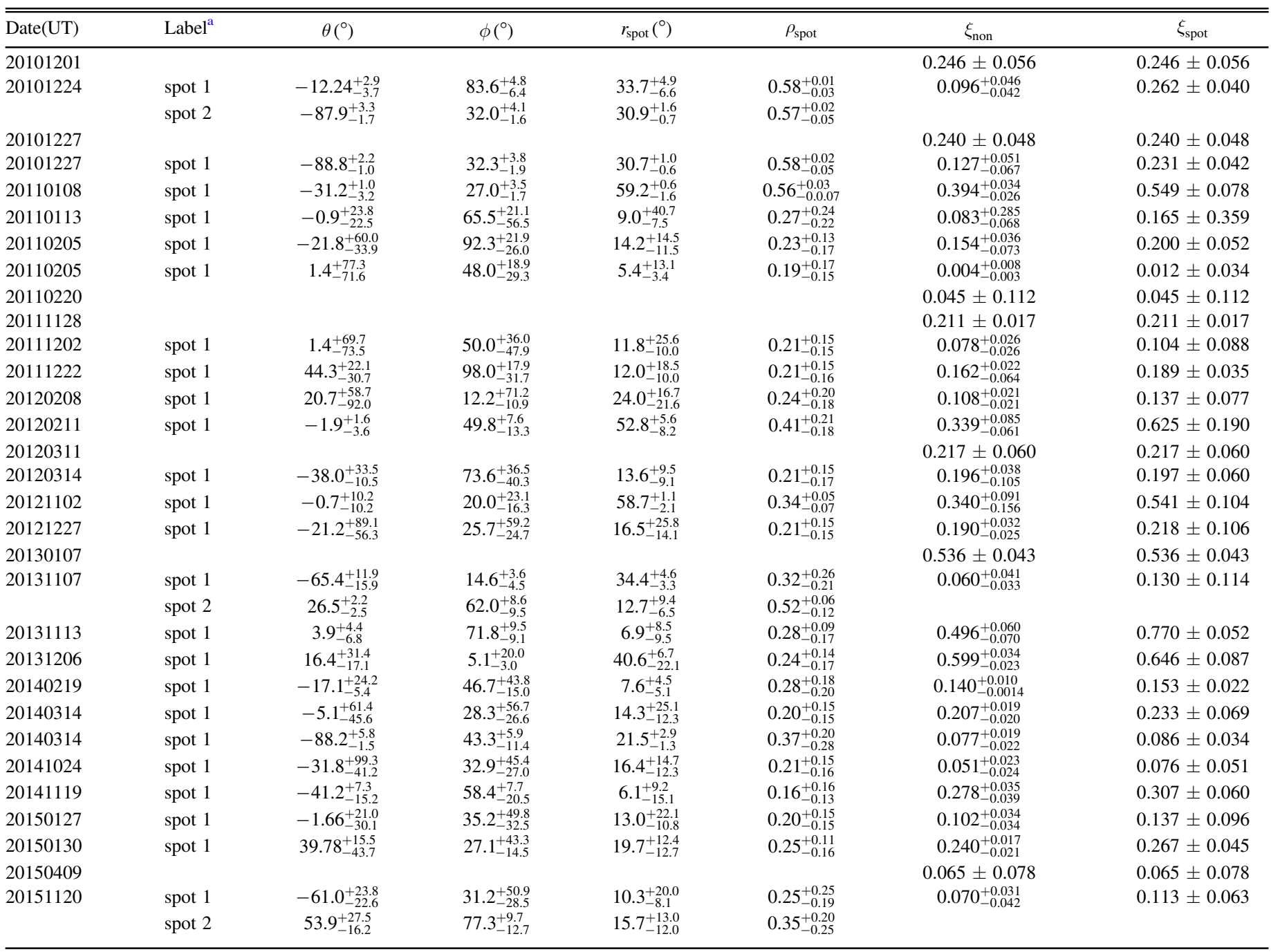

Note.

${ }^{\text {a }}$ Label occulted spots.

periodic TTVs induced by gravitational perturbation from another body based on these available data.

\subsection{Final Results}

In this subsection, we adopt a similar strategy to Section 5.1, namely combining 19 available light curves, which subtract the distortions by spots, with the radial velocity curve to derive the physical parameters of HAT-P-20 by utilizing the MCMC technique of Collier Cameron et al. (2007), but fixing the orbital period to the above newly derived value. These light curves include the two light curves of Bakos et al. (2011), three of Granata et al. (2014), one of Baştürk et al. (2015), our 12 light curves, and one derived by analyzing the raw photometric images from Mark Salisbury with PSF photometry before conducting aperture photometry and our standard procedure. Note that several transit light curves directly collected from the ETD are observed without a filter besides the ones obtained by Paul Benni and Alberto Villa, for which it is difficult to accurately estimate the limb-darkening coefficients. However, the limb-darkening coefficients need to be fixed when using the MCMC code of Collier Cameron et al. (2007) to model a transit light curve. Furthermore, the transit light curves directly collected from the ETD do not implement any systematic error correction. Therefore, these light curves are not included in the analysis of system parameters. By means of the MCMC code of Collier Cameron et al. (2007), we generate several chains to ensure the convergence of the solution and derive the optimal solution. The final solution of the physical parameters of HAT$\mathrm{P}-20$ is tabulated in Table 5.

\section{DISCUSSION}

Based on new photometric data and published photometric and radial velocity data, we have refined the physical parameters of transiting exoplanetary system HAT-P-20. Our new parameters are in good agreement with those of Bakos et al. (2011) with the exception of masses and radii of the host star and planet, and thus the bulk density of planet. We find that our measurements of $M_{p}$ and $R_{p}$ are larger than those of Bakos et al. (2011; see their Table 6 values: $M_{*}=0.756 \pm 0.028 M_{\odot} ; R_{*}=0.694 \pm 0.021 M_{\odot} ;$ $\left.M_{p}=7.246 \pm 0.187 M_{\mathrm{Jup}} ; \quad R_{p}=0.867 \pm 0.033 R_{\mathrm{Jup}}\right)$. Nonetheless, the measurements of both parameters (e.g., $M_{p}=$ $\left.7.191 M_{\mathrm{Jup}} ; R_{P}=0.888 R_{\mathrm{Jup}}\right)$ will be consistent with those of 


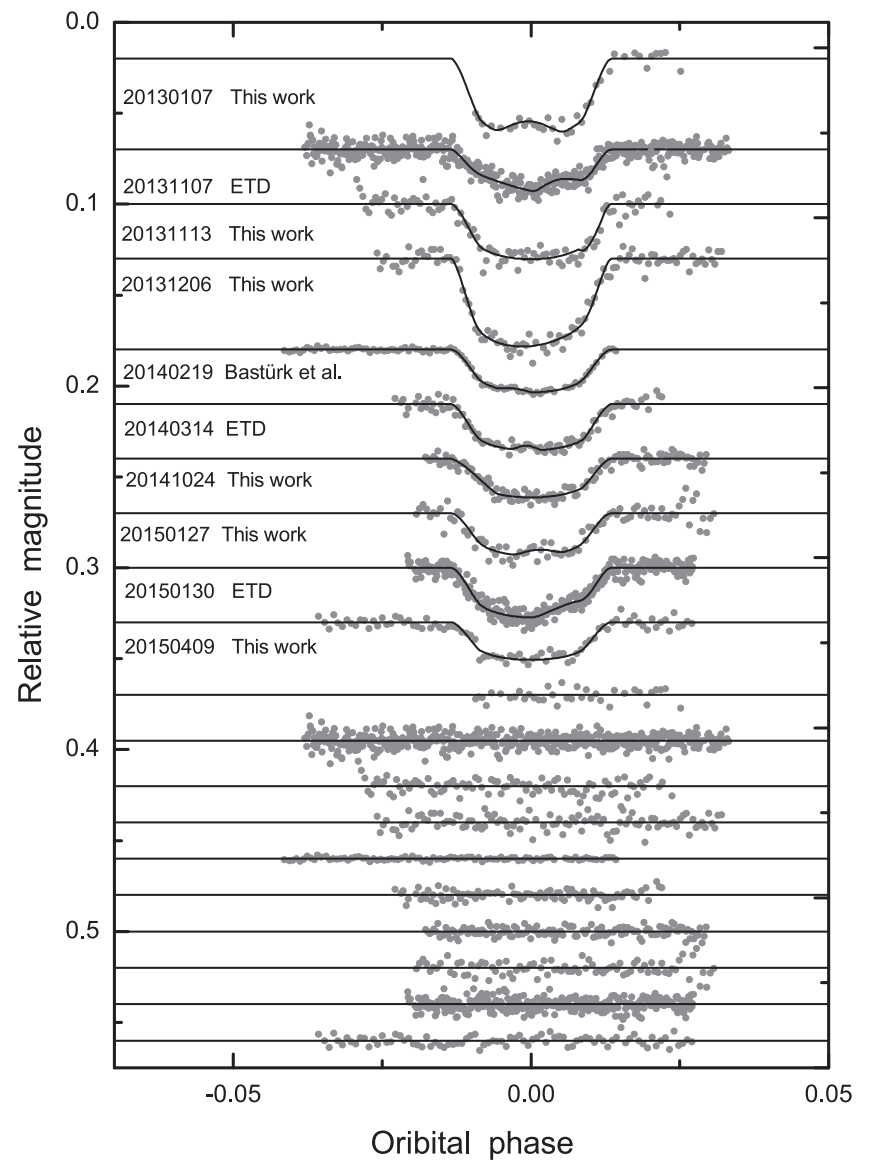

Figure 4. Model fittings of transit light curves of HAT-P-20 with STMT and relative residuals.

Bakos et al. (2011) when we adopt the mass and radius of HATP-20 derived by Bakos et al. (2011) from interpolating the stellar evolution model grids with the scaled semimajor axis of planetary orbit with stellar radius $a / R_{*}$, effective temperature $T_{\text {eff, }}$ and metallicity $\left[\mathrm{F}_{e} / \mathrm{H}\right]$ of the star. It is known that some K-dwarfs appear to be larger by $5 \%$ in radius than what is predicted by standard stellar models (Hoxie 1973; Popper 1997; Carter et al. 2011; Zhou et al. 2014; Maxted et al. 2015). This radius anomaly is correlated with the rotation rate of the star, but also shows some dependence on the mass and metallicity of the star (LópezMorales 2007; Spada et al. 2013). The dependence on rotation is thought to be the result of the increase in magnetic activity of rapidly rotating stars. Magnetic activity can affect the structure of a star by producing a high coverage of starspots, which changes the boundary conditions at the surface of the star, or by reducing the efficiency of energy transport by convection (Feiden \& Chaboyer 2014; Feiden 2015; Maxted et al. 2015). Therefore, it is apparent that our larger values derived from utilizing the empirical calibration of Enoch et al. (2010), obtained from observations of detached eclipsing binary systems, are possibly due to the effect of magnetic activity of HAT-P-20 on its own evolution, which is often not considered in most stellar evolution models.

Through the analysis of mid-transit times derived by modeling with the normal model and STMT, we obtain a more accurate orbital period of HAT-P-20b. Furthermore, we confirm Granata et al. (2014)'s hypothesis that stellar activity is responsible for the measured deviations of transit times based on a homogeneous study of spots and transit events of HAT-P20. However, sources of systematic noise in transit light curves

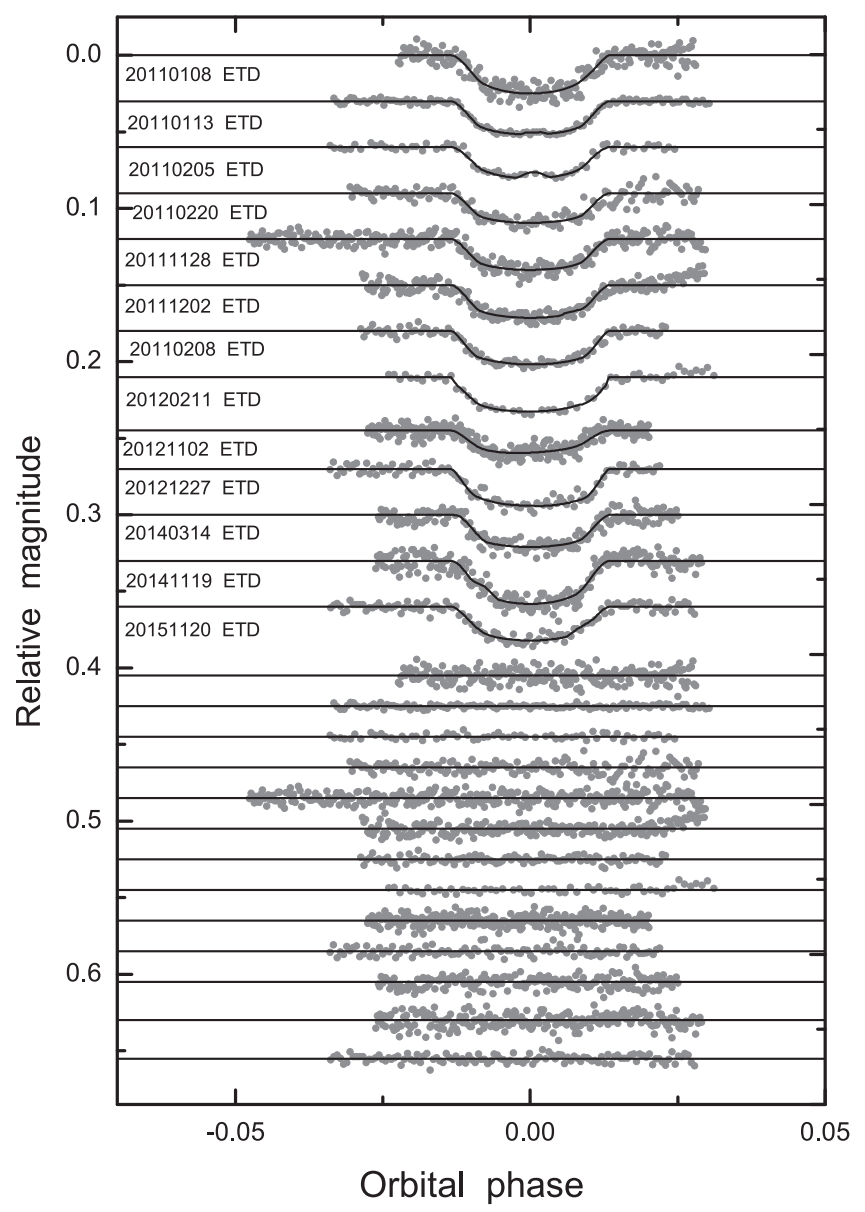

Figure 5. Model fittings of transit light curves of HAT-P-20 with STMT and relative residuals.

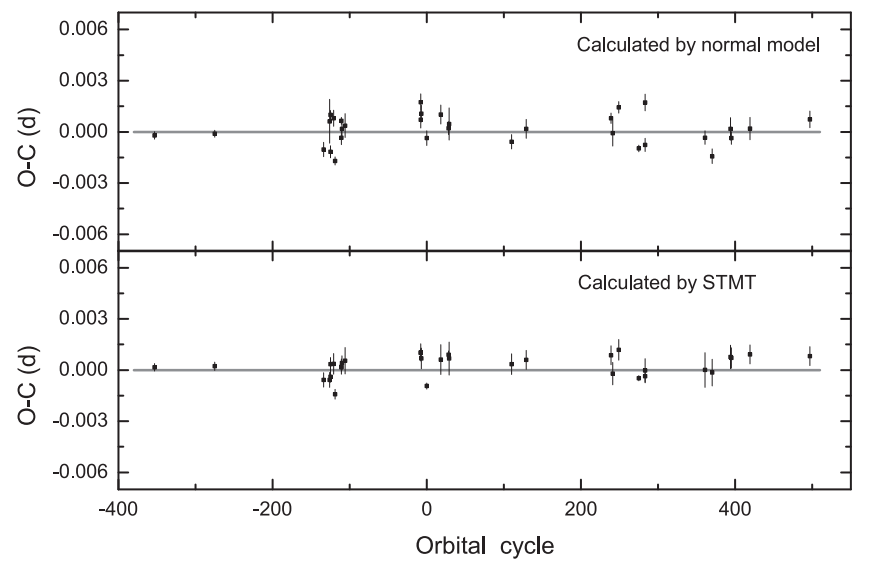

Figure 6. $(O-C)$ results based on mid-transit times calculated using the normal model and STMT.

are not considered. These are induced by the fluctuation of the flux contribution from the nearby companion along with the variation of seeing. Thus some sizeable, systematic distortions induced by the variation of seeing are possibly diagnosed as signals of occulted starspots. For instance, one pair of transit light curves (e.g., two transit light curves observed on 2010 December 27 by utilizing the YO- $1 \mathrm{~m}$ and HKNEAC- $0.5 \mathrm{~m}$ telescopes), which were observed for the same transit event by different observers using different equipment on two sites, show apparent evidence for a different number of spot crossing 


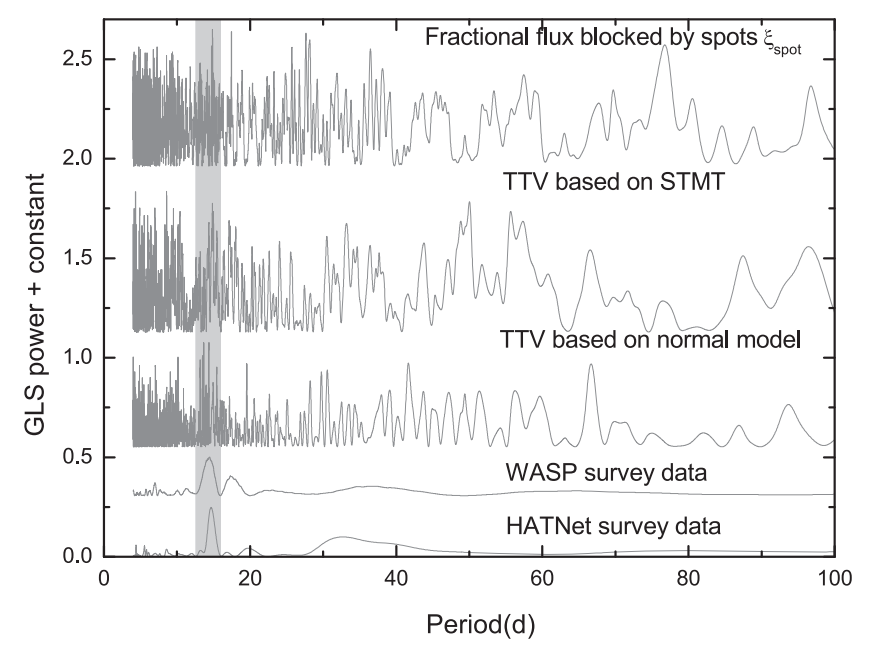

Figure 7. GLS periodagram of (bottom to top) HATNet survey data and WASP survey data of HAT-P-20, TTVs of HAT-P-20b based on the normal and spotted model, and the fractional flux of HAT-P- $20 \xi_{\text {spot }}$ blocked by starspots.

Table 5

Physical Parameters of HAT-P-20 Derived Using the MCMC Technique

\begin{tabular}{lcl}
\hline \hline Parameter & This work & Note \\
\hline$T_{0}\left(\mathrm{BJD}_{\mathrm{TDB}}\right.$ & 5917.64431 & \\
$-2450000)$ & \pm 0.00006 & Transit epoch \\
$P($ day $)$ & 2.8753172 & \\
& \pm 0.0000003 & Orbital period \\
$\Delta F$ & $0.0173 \pm 0.0002$ & Planet/star area ratio \\
$T_{14}($ day $)$ & $0.0781 \pm 0.0004$ & Transit duration \\
$b$ & $0.686_{-0.010}^{+0.009}$ & Impact parameter \\
$K_{1}\left(\mathrm{~m} \mathrm{~s}^{-1}\right)$ & $1247 \pm 6.3$ & Semi-amplitude of \\
& & radial velocity curve \\
$\gamma\left(\mathrm{m} \mathrm{s}^{-1}\right)$ & $-38 \pm 36$ & Centre-of-mass velocity \\
$e$ & $0.0136_{-0.0036}^{+0.0042}$ & Orbital eccentricity \\
$\omega($ degree $)$ & $330_{-18}^{+27}$ & Argument of periastron \\
$i($ degree $)$ & $86.3 \pm 0.1$ & Orbital inclination \\
$a($ au $)$ & $0.03671 \pm 0.00027$ & Orbital separation \\
$M_{*}\left(M_{\odot}\right)$ & $0.798 \pm 0.018$ & Stellar mass \\
$R_{*}\left(R_{\odot}\right)$ & $0.744 \pm 0.011$ & Stellar radius \\
$\rho_{*}\left(\rho_{\odot}\right)$ & $1.94 \pm 0.07$ & Stellar mean density \\
$M_{p}\left(M_{\text {Jup }}\right)$ & $7.59 \pm 0.12$ & Planet mass \\
$R_{p}\left(R_{\text {Jup }}\right)$ & $0.952 \pm 0.017$ & Planet radius \\
$\rho_{p}\left(\rho_{\text {Jup }}\right)$ & $8.80_{-0.43}^{+0.46}$ & Planet mean density \\
$T_{e q}(\mathrm{~K})$ & $996 \pm 19$ & Planet temperature \\
\hline & &
\end{tabular}

events. Underestimation of this kind of noise will thus underestimate the actual uncertainty of the mid-transit time $T_{c}$. This issue deteriorates for observations by utilizing smallsize telescopes with less spatial resolution. Furthermore, longterm variation of the seeing during these observations of transit events should be random and thus the joint distribution of the perturbation to $T$ caused by the issue should also be random. Therefore, it is this issue that probably produces relatively significant, non-periodic TTVs of HAT-P-20b (e.g., $\chi_{v}^{2}$ up to 4.09 for linear ephemeris).

From the above analysis, it should be noted that some occulted spots detected with STMT are not real spots but

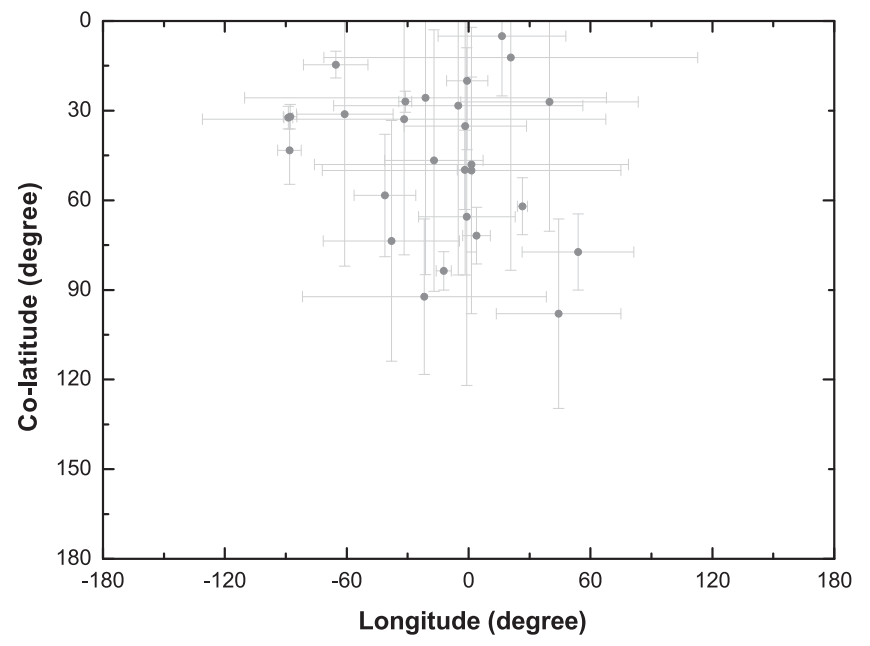

Figure 8. Distribution of occulted spots by HAT-P-20b in the stellar photosphere.

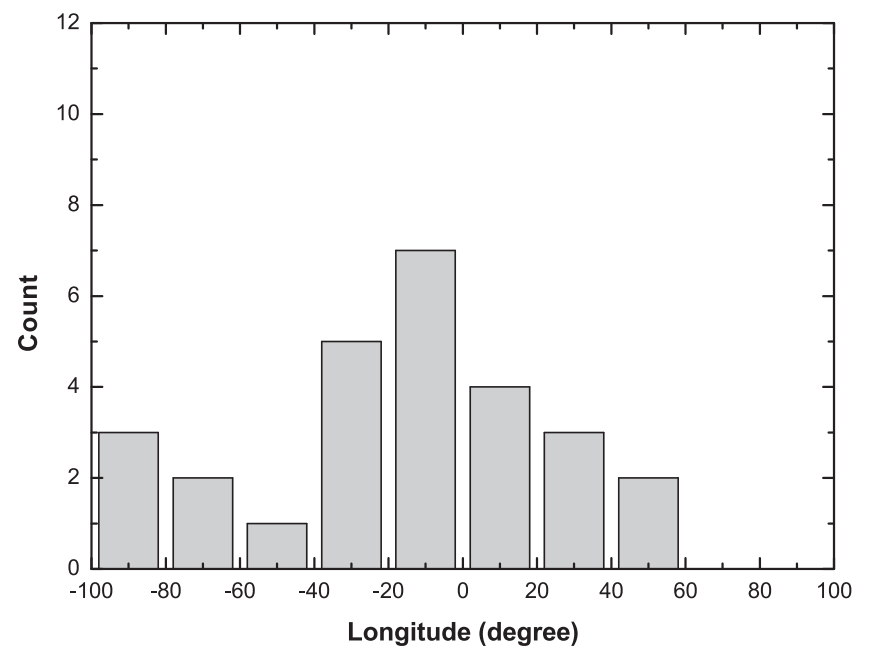

Figure 9. Histogram of the longitude of occulted spots by HAT-P-20b.

artifacts so as to fit significant systematic noise induced by instrumental defects and various seeing similar to the influence of spot crossing events on the transit light curves. Nonetheless, we have found the asymmetric distribution of occulted spots along the longitude $\theta$ (e.g., $-90^{\circ}<\theta \leqslant 60^{\circ}$; see Figures 8 and 9). As analyzed in the above section, the asymmetric distribution of occulted spots along with the longitude cannot be caused by artifacts mixed with real spots, since the joint distribution of these artifacts along with the longitude should be a random perturbation to $T_{c}$. Therefore, we claim that the asymmetric distribution of spots along the longitude derived with STMT is caused by a misaligned configuration between the planetary orbit and the spin of the host star in the transiting system HAT-P-20. Such a configuration could generate a positive deviation of the zero point between two ephemeris since the anomalies showing in the ingress of transit light curves will cause a positive perturbation to the mid-transit times derived when fitted with a normal model. Furthermore, flux contributions from the companion also infiltrate into the fractional deficit of photospheric flux $\xi_{\text {non }}$ derived with STMT when the transit light curves are acquired by employing the aperture photometry technique without removing the flux contribution from the companion. Namely, $\xi_{\text {non }}$ also plays the 
role of a dilution factor of the host star's flux caused by a nearby companion besides characterizing the flux blocked by non-occulted spots. Therefore, it is the presence of $\xi_{\text {non }}$ that makes homogeneous analysis of transit light curves of HAT-P20 obtained with different photometric techniques possible.

\section{SUMMARY}

From the above homogenous analysis for our new photometric observations, published light curves, and radial velocity curve, we have obtained new physical parameters of HAT-P20, which are consistent with those of Bakos et al. (2011), but which have higher accuracy. Through analyzing the distribution of spots occulted by HAT-P-20b, we deduce that the orbit of HAT-P-20b and the spin of the host star are misaligned. Through comparing the $(O-C)$ of mid-transit times derived with the normal and spotted model, a more accurate orbital period is derived, and the TTV signals detected by employing the normal model are found to be caused by spot crossing events rather than undetected bodies in the system.

We thank the staff of the $1 \mathrm{~m}$ and $2.4 \mathrm{~m}$ telescopes of Yunnan Observatories, the $0.5 \mathrm{~m}$ telescope of Ho koon Nature Education cum Astronomical Centre, and Observatori Ca l'Ou for supporting our observations. We also thank V. Granata, Özgür Baştürk, Paul Benni, Alberto Villa, and Mark Salisbury for generously providing us their data. We would like to thank M. B. N. Kouwenhoven and our anonymous referee for their insightful reading of our manuscript and their detailed comments, which improved our manuscript. This research has made use of the Keck Observatory Archive (KOA), which is operated by the W. M. Keck Observatory and the NASA Exoplanet Science Institute (NExScI), under contract with the National Aeronautics and Space Administration. This work is supported by National Natural Science Foundation of China through grants No. U1531121, No. 10873031 and No. 11473066.

\section{REFERENCES}

Agol, E., Steffen, J., Sari, R., \& Clarkson, W. 2005, MNRAS, 359, 567 Baraffe, I., Chabrier, G., \& Barman, T. 2008, A\&A, 482, 315

Baraffe, I., Chabrier, G., \& Barman, T. 2010, RPPh, 73, 016901

Barros, S. C. C., Boué, G., Gibson, N. P., et al. 2013, MNRAS, 430, 3032

Baştürk, Ö., Hinse, T. C., Özavci, I., et al. 2015, in ASP Conf. Ser. 496, Living

Together: Planets, Host Stars and Binaries, ed. S. M. Rucinski, G. Torres, \&

M. Zejda (San Francisco, CA: ASP), 370

Béky, B., Kipping, D. M., \& Holman, M. J. 2014, MNRAS, 442, 3686 Berdyugina, S. V. 2005, LRSP, 2, 8

Carter, J. A., Fabrycky, D. C., Ragozzine, D., et al. 2011, Sci, 331, 562

Charbonneau, P. 1995, AJ, 101, 309

Claret, A. 2000, A\&A, 359, 289

Claret, A. 2004, A\&A, 428, 1001

Collier Cameron, A., Pollacco, D., Street, R. A., et al. 2006, MNRAS, 373, 799

Collier Cameron, A., Wilson, D. M., West, R. G., et al. 2007, MNRAS, 380, 1230

Cubillos, P., Harrington, J., Madhusudhan, N., et al. 2014, ApJ, 797, 42

Daassou, A., Benkhaldoun, Z., Ait Moulay Larbi, M., \& Elazhari, Y. 2014, arXiv: 1407.1280
Deming, D., Knutson, H., Kammer, J., et al. 2015, ApJ, 805, 132

Désert, J.-M., Charbonneau, D., Demory, B.-O., et al. 2011, ApJS, 197, 14

Eastman, J., Gaudi, B. S., \& Agol, E. 2013, PASP, 125, 83

Eastman, J., Siverd, R., \& Gaudi, B. S. 2010, PASP, 122, 935

Eker, Z. 1999, NewA, 4, 365

Enoch, B., Collier Cameron, A., \& Horne, K. 2012, A\&A, 540, A99

Enoch, B., Collier Cameron, A., Parley, N. R., \& Hebb, L. 2010, A\&A, 516, A3

Feiden, G. A. 2015, in ASP Conf. Ser. 496, Living Together: Planets, Host Stars and Binaries, ed. S. M. Rucinski, G. Torres, \& M. Zejda (San Francisco, CA: ASP), 137

Feiden, G. A., \& Chaboyer, B. 2014, ApJ, 789, 53

Granata, V., Nascimbeni, V., Piotto, G., et al. 2014, AN, 335, 797

Gregory, P. C. 2011, MNRAS, 410, 94

Bakos, G. Á., Hartman, J., Torres, G., et al. 2011, ApJ, 742, 116

Herrero, E., Ribas, I., Jordi, C., et al. 2015, in Proc. XI Scientific Meeting of the Spanish Astronomical Society, Highlights of Spanish Astrophysics VIII, ed. A. J. Cenarro et al., 494

Holman, M. J., \& Murray, N. W. 2005, Sci, 307, 1288

Hoxie, D. T. 1973, A\&A, 26, 437

Kipping, D. M. 2009, MNRAS, 392, 181

Laloy, E., \& Vrugt, J. A. 2012, WRR, 48, 1526

López-Morales, M. 2007, ApJ, 660, 732

Mandel, K., \& Agol, E. 2002, ApJL, 580, L171

Maxted, P. F. L. 2016, A\&A, 591, A111

Maxted, P. F. L., Serenelli, A. M., \& Southworth, J. 2015, A\&A, 575, A36 Miralda-Escudé, J. 2002, ApJ, 564, 1019

Montalto, M., Boué, G., Oshagh, M., et al. 2014, MNRAS, 444, 1721

Nutzman, P. A., Fabrycky, D. C., \& Fortney, J. J. 2011, ApJL, 740, L10

Oshagh, M., Boisse, I., Boué, G., et al. 2013, A\&A, 549, A35

Oshagh, M., Santos, N. C., Boisse, I., et al. 2013, A\&A, 556, A19

Oshagh, M., Santos, N. C., Boisse, I., et al. 2015, European Physical Journal Web of Conferences, 101, 05003

Pál, A. 2012, MNRAS, 420, 1630

Pont, F., Gilliland, R. L., Moutou, C., et al. 2007, A\&A, 476, 1347

Popper, D. M. 1997, AJ, 114, 1195

Salz, M., Schneider, P. C., Czesla, S., \& Schmitt, J. H. M. M. 2015, A\&A, 576, A42

Sanchis-Ojeda, R., Winn, J. N., Holman, M. J., et al. 2011, ApJ, 733, 127

Sanchis-Ojeda, R., Winn, J. N., Marcy, G. W., et al. 2013, ApJ, 775, 54

Shkolnik, E., Walker, G. A. H., \& Bohlender, D. A. 2003, ApJ, 597, 1092

Shkolnik, E., Walker, G. A. H., Bohlender, D. A., Gu, P.-G., \& Kürster, M. 2005, ApJ, 622, 1075

Shkolnik, E. L. 2013, ApJ, 766, 9

Silva, A. V. R. 2003, ApJ, 585, 147

Silva-Valio, A. 2008, ApJL, 683, L179

Sozzetti, A., Torres, G., Charbonneau, D., et al. 2007, ApJ, 664, 1190

Spada, F., Demarque, P., Kim, Y.-C., \& Sills, A. 2013, ApJ, 776, 87

Stetson, P. B. 1987, PASP, 99, 191

Sun, L.-L., Gu, S.-H., Wang, X.-B., et al. 2015, RAA, 15, 117

Tamuz, O., Mazeh, T., \& Zucker, S. 2005, MNRAS, 356, 1466

Tan, H. B., Wang, X. B., Gu, S. H., \& Cameron, A. C. 2013, AcASn, 54, 527

ter Braak, C. J. F. 2006, Stat. Comput., 16, 239

Tregloan-Reed, J., Southworth, J., Burgdorf, M., et al. 2015, MNRAS, 450,1760

Tregloan-Reed, J., Southworth, J., \& Tappert, C. 2013, MNRAS, 428, 3671

Vrugt, J. A., ter Braak, C. J. F., Diks, C. G. H., et al. 2009, International Journal of Nonlinear Science \& Numerical Simulation, 10, 271

Wang, X.-B., Gu, S.-H., Collier Cameron, A., et al. 2013, RAA, 13, 593

Wang, X.-B., Gu, S.-H., Collier Cameron, A., et al. 2014, AJ, 147, 92

Wilson, O. C. 1968, ApJ, 153, 221

Wöllert, M., \& Brandner, W. 2015, A\&A, 579, A129

Wolter, U., Schmitt, J. H. M. M., Huber, K. F., et al. 2009, A\&A, 504, 561

Zechmeister, M., \& Kürster, M. 2009, A\&A, 496, 577

Zhou, G., Bayliss, D., Hartman, J. D., et al. 2014, MNRAS, 437, 2831 